\title{
A comprehensive profile of chemokines in the peripheral blood and vascular tissue of patients with Takayasu arteritis
}

\author{
Xiufang Kong ${ }^{1}$, Sifan Wu${ }^{1}$, Xiaojuan Dai ${ }^{1}$, Wensu Yu ${ }^{1}$, Jinghua Wang ${ }^{1}$, Ying Sun ${ }^{1}$, Zongfei Ji ${ }^{1}$, Lingying Ma ${ }^{1}$, \\ Xiaomin Dai ${ }^{1}$, Huiyong Chen ${ }^{1}$, Lili Ma ${ }^{1}$ and Lindi Jiang ${ }^{1,2^{*}}$ (D)
}

\begin{abstract}
Background: Takayasu arteritis (TAK) is a chronic granulomatous large vessel vasculitis with multiple immune cells involved. Chemokines play critical roles in recruitment and activation of immune cells. This study aimed to investigate chemokine profile in the peripheral blood and vascular tissue of patients with TAK.

Methods: A total of 58 patients with TAK and 53 healthy controls were enrolled. Chemokine array assay was performed in five patients with TAK and three controls. Chemokines with higher levels were preliminarily validated in 20 patients and controls. The validated chemokines were further confirmed in another group of samples with 25 patients and 25 controls. Their expression and distribution were also examined in vascular tissue from 8 patients and 5 controls. Correlations between these chemokines and peripheral immune cells, cytokines, and disease activity parameters were analyzed. Their serum changes were also investigated in these 45 patients after glucocorticoids and immunosuppressive treatment.

Results: Patients and controls were age and sex-matched. Twelve higher chemokines and 4 lower chemokines were found based on the chemokine array. After validation, increase of 5 chemokines were confirmed in patients with TAK, including CCL22, RANTES, CXCL16, CXCL11, and IL-16. Their expressions were also increased in vascular tissue of patients with TAK. In addition, levels of RANTES and IL-16 were positively correlated with peripheral $C D 3^{+} \mathrm{CD}^{+} \mathrm{T}$ cell numbers. Close localization of CCL22, CXCL11, or IL-16 with inflammatory cells was also observed in TAK vascular tissue. No correlations were found between these chemokines and cytokines (IL-6, IL-17, IFN- $\gamma$ ) or inflammatory parameters (ESR, CRP). No differences were observed regarding with these chemokines between active and inactive patients. After treatment, increase of CCL22 and decrease of RANTES and CXCL16 were found, while no changes were showed in levels of CXCL11 and IL-16.

Conclusions: CCL22, RANTES, CXCL16, CXCL11, and IL-16 were identified as the major chemokines involved in the recruitment of immune cells in the vascular tissue of patients with TAK. Additionally, the persistently high levels of CCL22, CXCL11, and IL-16 observed after treatment indicate their role in vascular chronic inflammation or fibrosis and demonstrate the need for developing more efficacious treatment options.
\end{abstract}

*Correspondence: zsh-rheum@hotmail.com

1 Department of Rheumatology, Zhongshan Hospital Affiliated to Fudan University, Shanghai, China

Full list of author information is available at the end of the article

\section{Background}

Takayasu arteritis (TAK) is a type of large vessel vasculitis that predominantly occurs in female patients of reproductive age ( $<40$ years) and leads to vascular thickness, stenosis, and even occlusion of the aorta and its branches [1]. TAK is more prevalent in Asian countries, such as original author(s) and the source, provide a link to the Creative Commons licence, and indicate if changes were made. The images or other third party material in this article are included in the article's Creative Commons licence, unless indicated otherwise in a credit line to the material. If material is not included in the article's Creative Commons licence and your intended use is not permitted by statutory regulation or exceeds the permitted use, you will need to obtain permission directly from the copyright holder. To view a copy of this licence, visit http://creativecommons.org/licenses/by/4.0/. The Creative Commons Public Domain Dedication waiver (http://creativeco mmons.org/publicdomain/zero/1.0/) applies to the data made available in this article, unless otherwise stated in a credit line to the data. 
China, Japan, Korea, and Turkey, than in western countries [2].

TAK is characterized by chronic inflammation, and multiple immune cells participate in the development of TAK. High numbers of helper T cells, follicular helper $\mathrm{T}$ cells, CD8 ${ }^{+} \mathrm{T}$ cells, CD14 ${ }^{+}$monocytes, and neutrophils cells have been found in the peripheral blood of patients with TAK [3]. In addition, various types of immune cells, such as macrophages, lymphocytes, and dendritic cells, have been observed in vascular lesions from patients with TAK [4]. Thus, the recruitment and activation of immune cells appear to be closely related with the development of vascular lesions in TAK.

Chemokines play critical roles in the recruitment of immune cells from the peripheral blood to injured tissue. For example, in giant cell arteritis (GCA), another large vessel vasculitis, CXCL9, CXCL10, and CXCL11 were reported to mediate macrophage infiltration in vascular lesions [5]. In TAK, vascular lesions originate from the vascular adventitia. The vascular adventitia is rich in vasa vasorum, which can transport peripheral immune cells to active vascular lesions in the early stage of lesion development [6]. In TAK patients, the levels of RANTES, CCL2, CCL20, CXCL8, and CXCL10 have been reported to be elevated in the peripheral blood, and their levels were correlated with disease activity $[7,8]$. However, the profile of chemokines in TAK has not been clearly elucidated.

Apart from the role of chemokines in the chemotaxis of immune cells from the peripheral blood to injured lesions, chemokines may also participate in the migration of immune cells through vascular layers in TAK. TAK lesions develop from the outside to the inside of vascular tissue [9]. In our previous study, we found that macrophage distributed differently in different stages [10]. Additionally, the expression of CCL2, the main chemokine associated with macrophages, was consistent with the distribution of macrophages [10]. These findings indicate that chemokine also plays an important role in the migration of immune cells within vascular tissue in TAK. However, the expression of chemokines in vascular tissues in TAK is still unclear.

The aim of this study is to explore the chemokine spectrum in the peripheral blood as well as vascular tissue from patients with TAK and investigate its potential role in the pathogenesis of TAK.

\section{Methods}

\section{Study population}

A total of 58 naive patients with TAK and 53 healthy controls were enrolled in this study from Zhongshan Hospital, Fudan University, Shanghai, China, between January 1 and December 31, 2020. Patients were diagnosed according to the 1990 American College of Rheumatology TAK classification criteria [11]. The study design is presented in Supplementary Fig. 1. Among the study participants, 50 patients with TAK and 48 healthy controls were selected for serum examination, while the remaining 8 patients and 5 controls were selected for vascular tissue examination. The patients for vascular tissue examination were scheduled to undergo surgery and were treatment-naive at the time of surgery. Vascular control samples were obtained from apparently healthy donors for heart (ascending aorta) or liver (abdominal aorta) transplantation. The study protocol was approved by the Ethics Committees of Zhongshan Hospital (B2016-168) and conformed to the ethical guidelines of the 1975 Declaration of Helsinki. Written informed consent was obtained from all the participants in this study.

\section{Data collection and clinical assessment}

Patient demographic and clinical data were recorded, including symptoms/signs, laboratory results, and imaging examination results. Laboratory parameters included inflammatory factors (erythrocyte sedimentation rate $[\mathrm{ESR}]$ and serum $\mathrm{C}$-reactive protein [CRP]) and immune cell numbers or proportions in the peripheral blood. These immune cells were detected by routine flow cytometry, including monocytes $\left(\mathrm{CD} 14^{+}\right)$, lymphocytes $\left(\mathrm{CD}^{+}\right), \mathrm{CD}^{+} \mathrm{T}$ cells $\left(\mathrm{CD}^{+}{ }^{+} \mathrm{CD} 4^{+}\right), \mathrm{CD} 8^{+} \mathrm{T}$ cells $\left(\mathrm{CD}^{+} \mathrm{CD}^{+}\right)$, B cells $\left(\mathrm{CD} 19^{+}\right)$, and natural killer (NK) cells $\left(\mathrm{CD} 56^{+}\right)$. A panel of fluorochrome-labeled monoclonal antibodies including CD3 (FITC, BioLegend), CD4 (PE-Cy7, BioLegend), CD8 (PE-Texas Red, BioLegend), CD14 (BV510, BioLegend), CD19 (PerCP/Cyanine5.5, BioLegend), and CD56 (APC, BioLegend) were used in flow cytometry and detected by BDFACSAriaIII. The gating strategy was shown in Supplementary Fig. 2. All the tests were conducted at the central laboratory of our center. Imaging examinations consisted of whole-body magnetic resonance angiography or computed tomographic angiography to assess vascular involvement.

Vascular types according to imaging findings were evaluated according to the imaging classification system created by Hata [12]. Disease activity was assessed based on the NIH activity criteria [13].

\section{Proteome profiler human chemokine array}

Of the 50 patients and 48 healthy controls for serum examination, five patients with TAK and three age- and gender-matched healthy controls were chosen for the chemokine array assay (ARY017; R\&D Systems Inc., Minneapolis). According to the instructions of the manufacturer, 31 chemokines were detected (Supplementary Fig. 3A). Results were expressed as the average signal (pixel density) of duplicate spots of each chemokine after 
the average background signal was subtracted. A 15\% higher or lower difference in the average signal between patients and controls was indicative of a differentially expressed chemokine.

\section{Enzyme-linked immunosorbent assay}

Based on the chemokine array results, 12 chemokines with higher levels in patients with TAK were preliminarily verified in 20 patients with TAK and 20 healthy controls by enzyme-linked immunosorbent assay (ELISA). These chemokines were CCL22, RANTES, CXCL16, CXCL11, IL-16, CCL1, CCL17, CCL19, CCL20, CXCL1, CX3CL1, and XCL1. After preliminary verification, five chemokines (CCL22, RANTES, CXCL16, CXCL11, and IL-16) with higher levels in TAK cases were further detected in the remaining 25 patients with TAK and 25 controls for further confirmation.

To clarify the effects of glucocorticoids and immunosuppressants on the expression of the chemokines, the serum levels of these five chemokines were also detected in the 45 patients with TAK after treatment. To investigate immune responses in the patients with TAK, the expression of relevant cytokines, including IL-6, IL-17, and IFN- $\gamma$ was also detected in the 45 patients (baseline and post-treatment) and 45 controls. Commercial ELISA kits purchased from Boster Bio (CA, USA) were used to detect these chemokines and cytokines.

\section{Immunofluorescence and immunohistochemistry}

To evaluate vascular infiltration upon vascular tissue, immunofluorescence for CD3 (ab16669, Abcam, MA, USA), CD19 (ab245235, Abcam, MA, USA), and CD68 (ab213363, Abcam, MA, USA) staining was performed. During this process, the deparaffination, rehydration, antigen retrieval, and blocking process were conducted as previously described [14]. During the staining process, CD68 antibody was firstly incubated at $4{ }^{\circ} \mathrm{C}$ overnight. On the 2nd day, after washing with PBS supplemented with $0.01 \%$ Tween-20, the corresponding secondary antibody was applied at room temperature for $1 \mathrm{~h}$. Then, Tyramide-CY3 was used to detect the primary antibody binding. Then, the second antigen retrieval process was conducted for $\mathrm{CD} 3$ staining. The staining process were same to the first antigen and detected by Tyramide-FITC. After this, the slides went through antigen retrieval process again. CD19 was finally detected by CY5 conjugated secondary antibody. At last, the slides were mounted with anti-fluorescence quenching agent containing DAPI.

For the immunohistochemistry analysis, primary antibodies against CCL22 (abs118802; Absin, Shanghai, China), RANTES (abs131231; Absin, Shanghai, China), CXCL16 (abs122925; Absin, Shanghai, China), CXCL11 (ab9955; Abcam, MA, USA), and IL-16 (ab207181;
Abcam, MA, USA), and their corresponding secondary antibodies were applied.

All the slides were digitally scanned using a 3DHISTECH scanning microscope, and images were viewed and selected using CaseViewer 2.4.0 (3DHISTECH Ltd., Hungary). In the quantification process, 10 views (about $0.15 \mathrm{~mm}^{2}$ ) upon $400 \mathrm{X}$ magnification within the greatest inflammatory infiltrates were selected from each slide. The average number of CD3 or CD19 or CD68-positive cells were calculated using Image and graded as +++ (the average number $>100 / 0.15 \mathrm{~mm}^{2}$ ), ++ (the average number $\left.50-100 / 0.15 \mathrm{~mm}^{2}\right),+$ (the average number $10-50 / 0.15 \mathrm{~mm}^{2}$ ), or - (the average number $<10 / 0.15 \mathrm{~mm}^{2}$ ). Chemokine expressions were semi-quantified by IHC profiler in ImageJ. The results were presented as negative $(-)$, weak positive $(+)$, moderate positive $(++)$, and strong positive $(+++)$.

\section{Evaluation of the clinical significance of the identified chemokines}

Distribution of the identified five chemokines (CCL22, RANTES, CXCL16, CXCL11, and IL-16) within inflammatory cells in vascular tissue and correlations between chemokine levels and immune cell $\left(\mathrm{CD}^{+}{ }^{+} \mathrm{T}\right.$ cell, $\mathrm{CD} 8^{+}$ $\mathrm{T}$ cell, $\mathrm{CD} 14^{+}$monocyte, $\mathrm{CD} 19^{+} \mathrm{B}$ cell, and $\mathrm{CD} 56^{+}$ NK cell) numbers in the peripheral blood were analyzed to investigate their potential role in immune cell recruitment. In addition, correlations between these chemokines and cytokines (IL-6, IL-17, and IFN- $\gamma$ ) were analyzed to explore their potential role in TAK immune response. Moreover, the levels of these five chemokines were compared between patients with active and inactive disease, and their correlations with inflammatory parameters (ESR and CRP) were examined to clarify their role in disease activity. Finally, changes in these chemokines were analyzed after glucocorticoids and immunosuppressive treatment.

\section{Statistical analysis}

Measurement data, such as chemokine levels, ESR, and CRP, were expressed as mean \pm standard deviation, while enumeration data, such as patients with active/inactive disease, were presented as frequencies and percentages. Student's $t$ test or paired $t$ test were used to compare data that was normally distributed, while Mann-Whitney test or Wilcoxon rank test were used to compare nonparametric data. In the correlation analysis, Pearson correlation analysis was performed to analyze normal distribution data, while Spearman correlation analysis was applied for non-parametric data. All statistical analyses were performed using SPSS version 20.0 (Chicago, IL, USA). Graphs were generated using GraphPad Prism 5 
(GraphPad Software Inc., USA). P values $<0.05$ were considered to indicate statistical significance.

\section{Results}

\section{Patient characteristics}

The patients with TAK and healthy controls selected for serum examination were matched for age (TAK: 34.44 \pm 13.25 years, control: $37.85 \pm 5.10$ years, $p=0.27$ ) and female to male ratio (TAK: 41:9, control: $36: 12, p=$ 0.40). The clinical characteristics of patients with TAK included in the serum examination are listed in Table 1. Among the 50 patients with TAK, 40 (80\%) had active disease at the time of enrollment. No significant differences were observed in the clinical characteristics $(p>0.05$ for all parameters, Table 1$)$ of patients $(n=$ $5)$ selected for chemokine screening and those selected for chemokine validation $(n=45)$.
The clinical characteristics of patients $(n=8)$ and controls $(n=5)$ selected for vascular tissue examination are listed in Supplementary Table 1. The main reasons for surgery were aortic regurgitation $(n=7)$ and renal artery occlusion $(n=1)$. Thus, ascending aortic specimens $(n=7)$ and a renal artery specimen $(n=1)$ were obtained. In addition, apparently normal specimens (control) of the ascending aorta $(n=3)$ and abdominal aorta $(n=2)$ were obtained from donors for heart transplantation $(n=3)$ and liver transplantation $(n=2)$.

\section{Increased expression of CCL22, RANTES, CXCL16, CXCL11,} and IL-16 in the peripheral blood of patients with TAK

The main functions of 31 chemokines in the array are listed in Supplementary Table 2. A representative image of the chemokine array results is shown

Table 1 Clinical characteristics of patients included in the serum examination

\begin{tabular}{|c|c|c|c|c|c|}
\hline \multirow[t]{2}{*}{ Clinical Parameters } & \multicolumn{4}{|l|}{ Patients with TAK } & \multirow[t]{2}{*}{ Healthy controls $(n=48)$} \\
\hline & Total $(n=50)$ & $\begin{array}{l}\text { For chemokine } \\
\text { screening }(n=5)\end{array}$ & $\begin{array}{l}\text { For chemokine } \\
\text { validation }(n=45)\end{array}$ & $p$ & \\
\hline \multicolumn{6}{|l|}{ General information } \\
\hline Gender ratio (F:M) & $41: 9$ & $5: 0$ & $36: 9$ & 057 & $36: 12$ \\
\hline Age at diagnosis (mean $\pm S D, y)$ & $34.40 \pm 13.25$ & $28.80 \pm 6.61$ & $35.07 \pm 13.69$ & 0.32 & / \\
\hline Disease duration (months) & $6.00(2.00-15.00)$ & $12.00(1.50-30.0 \mathrm{a} 0)$ & $6.00(2.00-12.00)$ & 0.68 & / \\
\hline Active disease status (n, \%) & $40(80.00)$ & $3(60)$ & $37(82.22)$ & 0.26 & / \\
\hline \multicolumn{6}{|l|}{ Clinical symptoms } \\
\hline Weakness (n, \%) & $11(22.00)$ & $2(40)$ & $9(20.00)$ & 0.30 & / \\
\hline Fever $(n, \%)$ & $6(12.00)$ & $1(20)$ & $5(11.11)$ & 0.49 & / \\
\hline Hypertension (n, \%) & $14(28.00)$ & $1(20)$ & $13(28.89)$ & 1.00 & / \\
\hline Claudication (n, \%) & $2(4.00)$ & $0(0)$ & $2(4.44)$ & 1.00 & / \\
\hline Chest distress (n, \%) & $9(18.00)$ & $0(0)$ & $9(20.00)$ & 0.57 & / \\
\hline Dizziness (n, \%) & $24(48.00)$ & $3(60)$ & $21(46.67)$ & 0.66 & / \\
\hline Pulselessness/weak pulse (n, \%) & $17(34.00)$ & $2(40)$ & $15(33.33)$ & 1.00 & / \\
\hline Vascular bruits (n, \%) & $17(34.00)$ & $3(60)$ & $14(31.11)$ & 0.32 & / \\
\hline \multicolumn{6}{|l|}{ Lab results } \\
\hline Hemoglobin (mean $\pm S D, g / L)$ & $119.41 \pm 15.20$ & $115.00 \pm 13.93$ & $119.62 \pm 15.35$ & 0.50 & $132.69 \pm 13.42$ \\
\hline WBC $\left(\right.$ mean $\left.\pm S D, 10^{9} / \mathrm{L}\right)$ & $8.99 \pm 5.87$ & $9.51 \pm 2.47$ & $8.95 \pm 6.10$ & 0.97 & $6.23 \pm 2.06$ \\
\hline $\mathrm{PLT}\left(\right.$ mean $\left.\pm \mathrm{SD}, 10^{9} / \mathrm{L}\right)$ & $293.47 \pm 93.70$ & $346.25 \pm 140.67$ & $288.78 \pm 89.18$ & 0.12 & $258.46 \pm 61.55$ \\
\hline $\mathrm{ESR}(\mathrm{mean} \pm \mathrm{SD}, \mathrm{mm} / \mathrm{H})$ & $44.18 \pm 30.18$ & $59.50 \pm 31.69$ & $42.82 \pm 30.04$ & 0.29 & / \\
\hline $\mathrm{CRP}($ mean $\pm \mathrm{SD}, \mathrm{mg} / \mathrm{L})$ & $24.40 \pm 26.69$ & $32.13 \pm 26.18$ & $23.71 \pm 26.92$ & 0.55 & / \\
\hline IL-6 (mean \pm SD, pg/ml) & $11.80 \pm 10.26$ & $12.54 \pm 10.28$ & $11.71 \pm 10.38$ & 0.87 & / \\
\hline TNF-a (mean \pm SD, pg/ml) & $8.94 \pm 7.99$ & $4.93 \pm 0.68$ & $9.42 \pm 8.33$ & 0.29 & / \\
\hline \multicolumn{6}{|l|}{ Imaging type } \\
\hline 1 & $13(26.00)$ & $4(80)$ & $9(20.00)$ & 0.028 & I \\
\hline$\| a$ & $2(4.00)$ & $0(0)$ & $2(4.44)$ & & / \\
\hline$\| \mathrm{b}$ & $8(16.00)$ & $0(0)$ & $8(17.78)$ & & / \\
\hline III & $1(2.00)$ & $0(0)$ & $1(2.22)$ & & / \\
\hline IV & $5(10.00)$ & $1(20)$ & 4 (8.89) & & / \\
\hline V & $22(48.89)$ & $0(0)$ & $22(46.67)$ & & / \\
\hline
\end{tabular}


in Supplementary Fig. 3A, and the combined results are shown in Supplementary Fig. 3B. Among the 31 chemokines, the average signal of 12 chemokines was over $15 \%$ higher in patients with TAK than in the healthy controls (Supplementary Fig. 3C a-l): CCL22, RANTES, CXCL16, CXCL11, IL-16, CCL1, XCL1, CX3CL1, CXCL1, CCL17, CCL19, and CCL20. In contrast, the average signal of four chemokines (CXCL10, CXCL7, CCL18, and CXCL4) was 15\% lower in patients with TAK than in the healthy controls (Supplementary Fig. $3 \mathrm{C} \mathrm{m}-$ p).

The 12 chemokines with significantly higher expression in patients with TAK were priliminarlily validated in 20 patients with TAK and 20 healthy controls. The results showed that the levels of five chemokines were significantly higher in patients with TAK than in the healthy controls (CCL22: $p<0.0001$, RANTES: $p<0.0001$, CXCL16: $p=0.01$, CXCL11: $p=0.04$, IL-16: $p=0.04$ ) (Fig. 1A). However, no significant differences were found in the other seven chemokines (CCL1, XCL1, CX3CL1, CXCL1, CCL17, CCL19, and CCL20: $p>0.05$ for all) (Fig. 1B).

To confirm these five chemokines with higher levels in patients with TAK, their levels were further detected and analyzed in another 25 controls and patients with TAK. The results also confirmed that these five chemokines were significantly higher in the patients with TAK (CCL22: $p<0.0001$, RANTES: $p<0.0001$, CXCL16: $p$ $<0.0001$, CXCL11: $p<0.01$, IL-16: $p=0.03$ ) (Fig. 1C). The data indicate that these five chemokines, CCL22, RANTES, CXCL16, CXCL11, and IL-16, might play a role in TAK development.

Increased expression of CCL22, RANTES, CXCL16, CXCL11, and IL-16 in vascular tissue and their relationships with vascular and peripheral immune cell numbers in patients with TAK

The immunofluorescence staining showed different grades of inflammatory infiltrates $\left(\mathrm{CD}^{+}\right.$or $\mathrm{CD}^{+} 9^{+}$or $\mathrm{CD}^{+} 8^{+}$cells) in vascular adventitia (Supplementary Table 1 and Supplementary Fig. 4). The expressions of these five chemokines were also increased in TAK vascular lesions (Supplementary Table 1 and Fig. 2A). Specifically, CCL22 was expressed in a weak positive to moderate positive grade $(+\sim++)$ and mainly detected at sites with inflammatory cell infiltration (Fig. 2A: CCL22); RANTES was weak positively $(+)$ expressed in vascular adventitia, which was not necessarily related to inflammatory cells (Fig. 2A: RANTES); CXCL16 was weak to strong positively $(+\sim+++)$ expressed and predominantly detected in microvessel walls in the adventitia (Fig. 2A: CXCL16); CXCL11 was moderate to strong positively expressed $(++\sim+++)$ in microvessel walls as well as lesions infiltrated with inflammatory cells (Fig. 2A: CXCL11); and IL-16 expression was also in a moderate to strong positive grade $(++\sim+++)$ and closely related with the distribution of inflammatory cells (Fig. 2A: IL-16).

Based on the observations above, the correlations between increased chemokine levels and peripheral immune cells were further analyzed among 45 patients (Fig. 2B). Compared with the findings in TAK tissue, the relationship between the chemokines and peripheral immune cells $\left(\mathrm{CD}^{+}{ }^{+} \mathrm{CD} 4^{+} \mathrm{T}\right.$ cells, $\mathrm{CD}^{+}{ }^{+} \mathrm{CD} 8^{+} \mathrm{T}$ cells, $\mathrm{CD} 19^{+} \mathrm{B}$ cells, $\mathrm{CD} 56^{+} \mathrm{NK}$ cells, and $\mathrm{CD} 14^{+}$monocytes) exhibited a different pattern. Surprisingly, no correlations were found between CCL22 and immune cell numbers in peripheral blood (Fig. 2B a1-a5). Similarly, no correlations were observed between CXCL16 and immune cell numbers either (Fig. 2B c1-c5). However, RANTES was weakly correlated with $\mathrm{CD} 3^{+} \mathrm{CD} 4^{+} \mathrm{T}$ cell number (rho

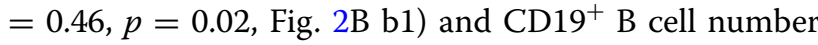
(rho $=0.40, p=0.04$, Fig. $2 \mathrm{~B}$ b3), but it was not correlated with the other cell types (Fig. 2B b2, b4, b5). With regard to CXCL11, it was weakly correlated with CD19 ${ }^{+}$ cell number (rho $=0.50, p=0.008$, Fig. $2 \mathrm{~B} \mathrm{~d} 3$ ), but not with the number of the other immune cells (Fig. 2B d1, $\mathrm{d} 2, \mathrm{~d} 4$, \& d5). Interestingly, IL-16 was weakly correlated with $\mathrm{CD} 3^{+} \mathrm{CD}^{+} \mathrm{T}$ cell number (rho $=0.46, p=0.02$, Fig. $2 \mathrm{~B}$ e1), but not with the number of other types of cells (Fig. 2B e2-e5).

These results indicate that while the chemokine expression was localized with immune cell numbers in vascular tissue, the correlations of these chemokines with immune cell numbers in the peripheral blood were limited.

\section{Correlation between increased chemokine levels and inflammatory cytokine levels in the peripheral blood of patients with TAK}

Higher levels of IL-6 $(p<0.001)$ and IL-17 $(p=0.004)$ in patients with TAK in contrast to the healthy controls (Supplementary Fig. 5A \& B). However, IFN- $\gamma$ levels were lower in the patients than in the healthy controls $(p<$ 0.001, Supplementary Fig. 5C). These data indicate that higher chemokine levels were accompanied with increase in the levels of IL- 6 and IL-17, which are the two main inflammatory cytokines involved in the development of TAK. The correlation analysis between these chemokines and cytokines (Supplementary Fig. 5D) indicated a weak correlation between RANTES and IL-6 levels (rho = $0.41, p=0.02$, Supplementary Fig. 5D b1). However, no correlations were found between the other chemokines and IL-6 or IL-17 levels (Supplementary Fig. 5D). 


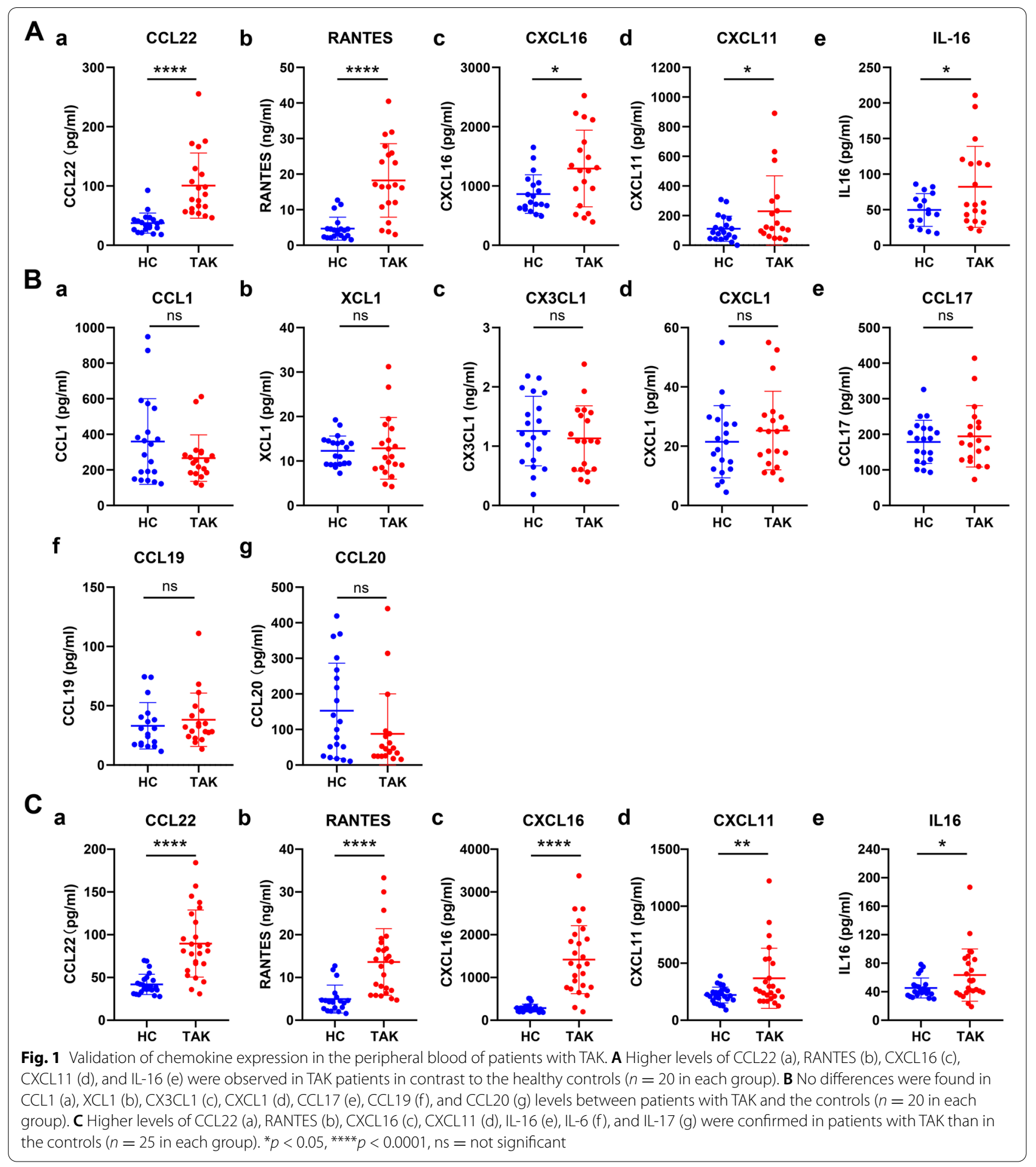

Correlations between chemokine levels and disease status Unfortunately, no differences were found in the levels of five chemokines between active and inactive patients (Supplementary Fig. 6A a-e), and no correlations were observed between these chemokines and the CRP (Supplementary Fig. 6B) or ESR (Supplementary Fig. 6C) levels either.

\section{Correlations between individual chemokine levels}

The results showed that levels of CCL22, CXCL16, and IL-16 were mutually correlated. In particular, CCL22 was positively correlated with CXCL16 $(r=0.57, p<$ 0.0001, Supplementary Fig. 7A) and IL-16 (rho $=0.57$, $p<0.0001$, Supplementary Fig. 7B), and IL-16 was also 


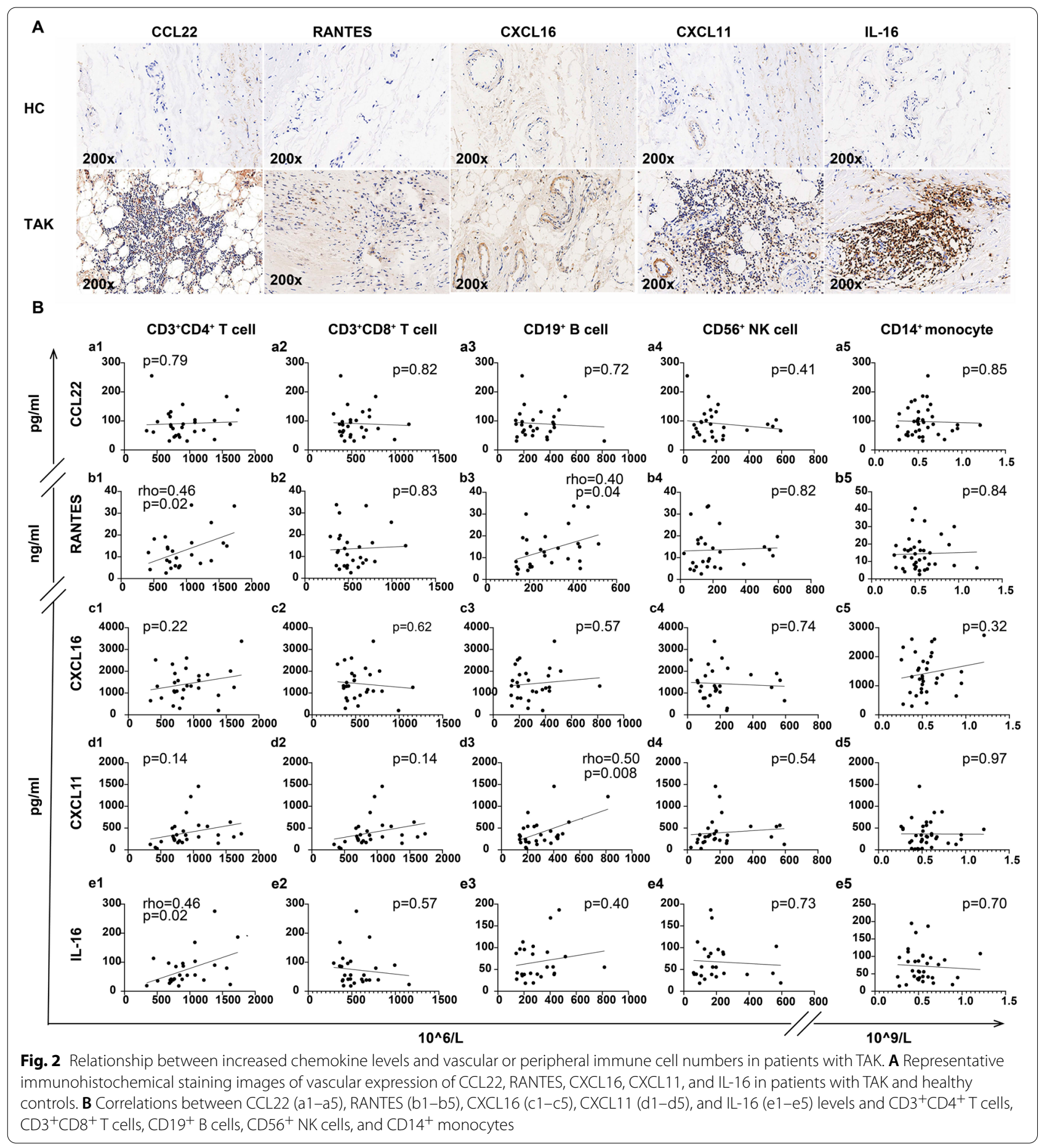

positively related with CXCL16 (rho $=0.40, p=0.005$, Supplementary Fig. 7C).

\section{Effect of immunosuppressive treatment on the identified chemokines and TAK-related cytokines}

The treatment included glucocorticoids combined with leflunomide (20 patients, 44.44\%), cyclophosphamide
(15 patients, 33.33\%), tocilizumab (8 patients, $17.78 \%$ ), or methotrexate ( 2 patients, $4.44 \%$ ). The median treatment duration was 6 (interquartile range: 5-6) months. After treatment, 14 (31.11\%) patients had active disease according to their NIH score.

After treatment, a further increase was observed in CCL22 levels $(85.66(52.39,114.64)$ vs. 111.20 
(51.71, 160.67), $p=0.03$, Fig. 3A), while there was a significant decrease in RANTES (14.68 \pm 9.35 vs. $9.43 \pm 5.90, p=0.001)$ and CXCL16 $(1381.17 \pm 743.26$ vs. $1083.03 \pm 447.39, p=0.02$ ) (Fig. 3B). However, no differences were observed in the levels of CXCL11 (328.35 $(168.55,535.43)$ vs. $352.13(271.29,604.85), p=0.31$, Fig. 3C a) and IL-16 (56.95 (39.24, 96.19) vs. 70.25 (48.60, $126.25), p=0.11$, Fig. $3 \mathrm{C}$ b). No changes were found in the levels of the three TAK-related cytokines (IL-6: 32.57 (25.46, 46.73) vs. 27.58 (22.34, 42.58), $p=0.08$, IL-17: $86.97(58.30,130.54)$ vs. $74.01(45.00,126.88), p=0.48$,

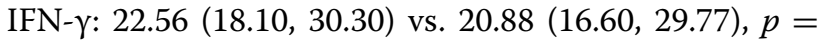
0.39 , Fig. $3 \mathrm{C} \mathrm{c}-\mathrm{e})$ after treatment.

We found that changes in the examined chemokines after treatment differed across individual patients. Therefore, immune cell numbers, ESR, CRP, and cytokines changes in the peripheral blood after treatment were compared between patients with increased and decreased levels of each chemokine. However, no differences were observed in these indicators between the two groups (data not shown). Correlations between the changes in these chemokines were also analyzed, and the results indicated similar trends in the changes in CCL22, CXCL16, and IL-16 levels after treatment (Supplementary Fig. 7D-F).

A summary of the characteristics of these five chemokines in patients with TAK is shown in Table 2. A proposed model illustrating chemokines discovered in the present study and their potential role in the pathogenesis of TAK was shown in Fig. 4.

\section{Discussion}

The present study demonstrates that the chemokines CCL22, RANTES, CXCL16, CXCL11, and IL-16 are elevated in the peripheral blood as well as vascular tissue of patients with TAK, and expression of CCL22, CXCL11, and IL-16 are distributed in vascular inflammatory lesions. These phenomena indicated that they may play a role in the recruitment of immune cells in vascular lesions of TAK. Further, after routine glucocorticoids and immunosuppressant treatment, RANTES and CXCL16 are decreased, while CCL22, CXCL11, and IL-16 present

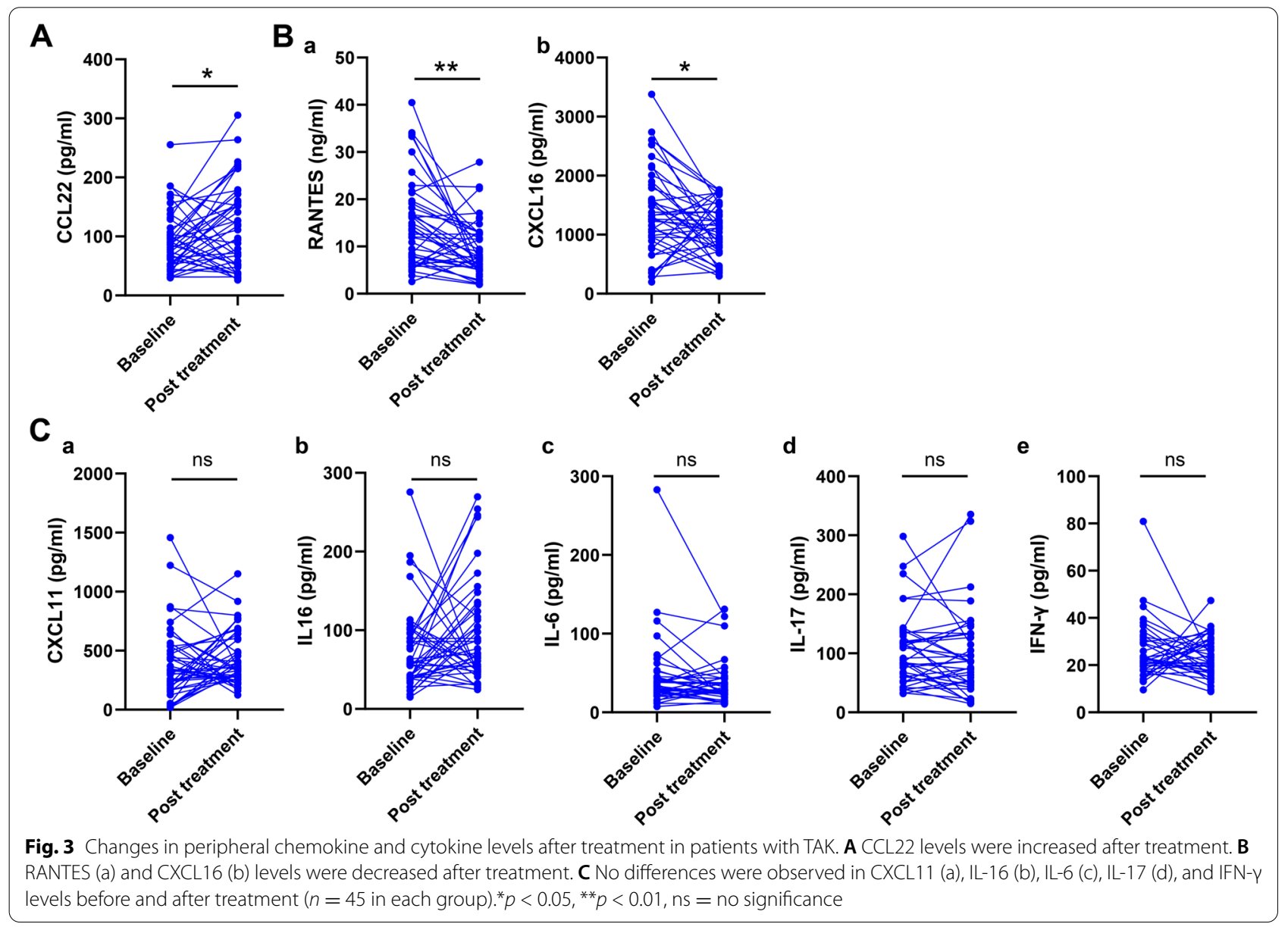




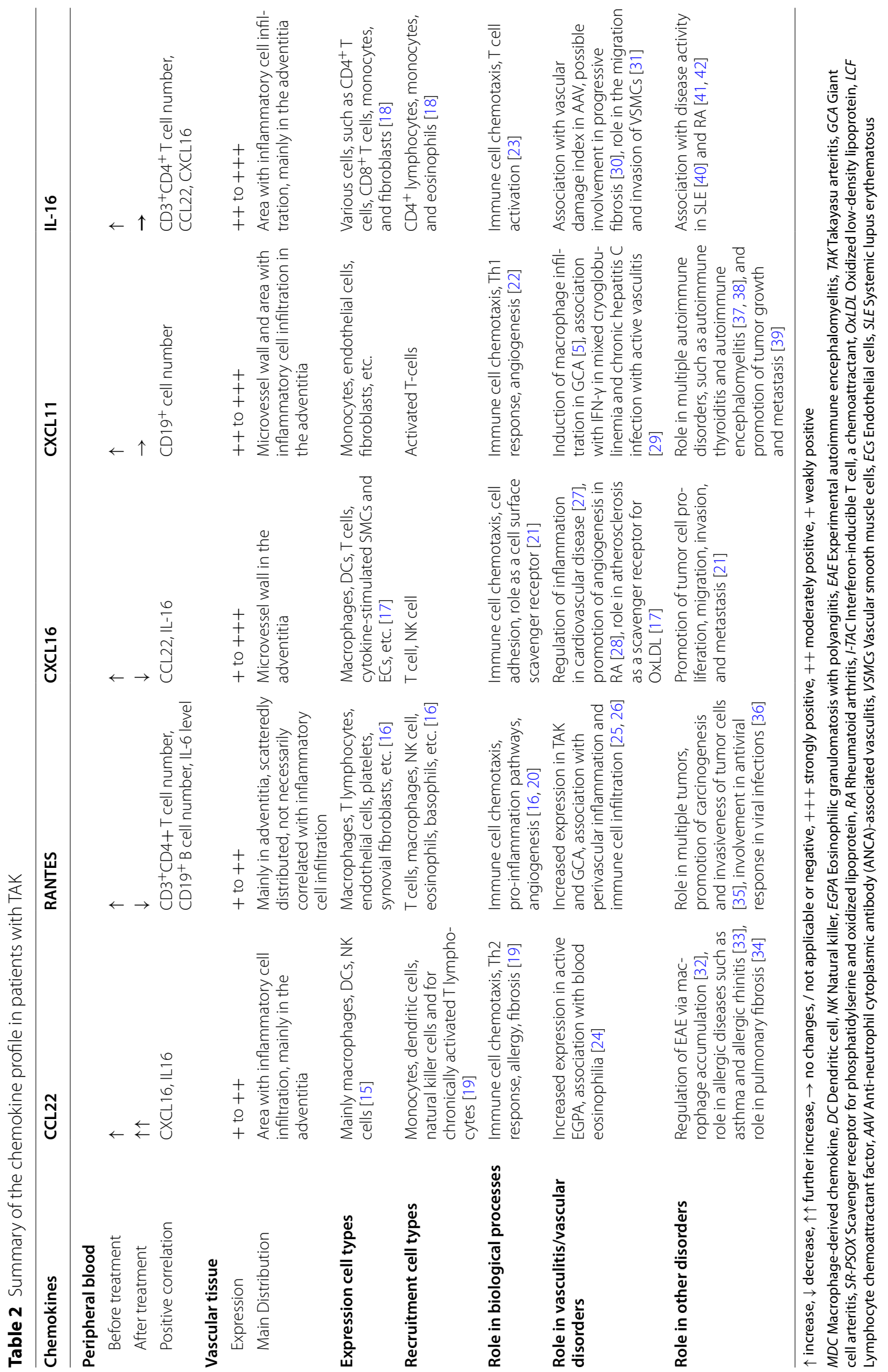




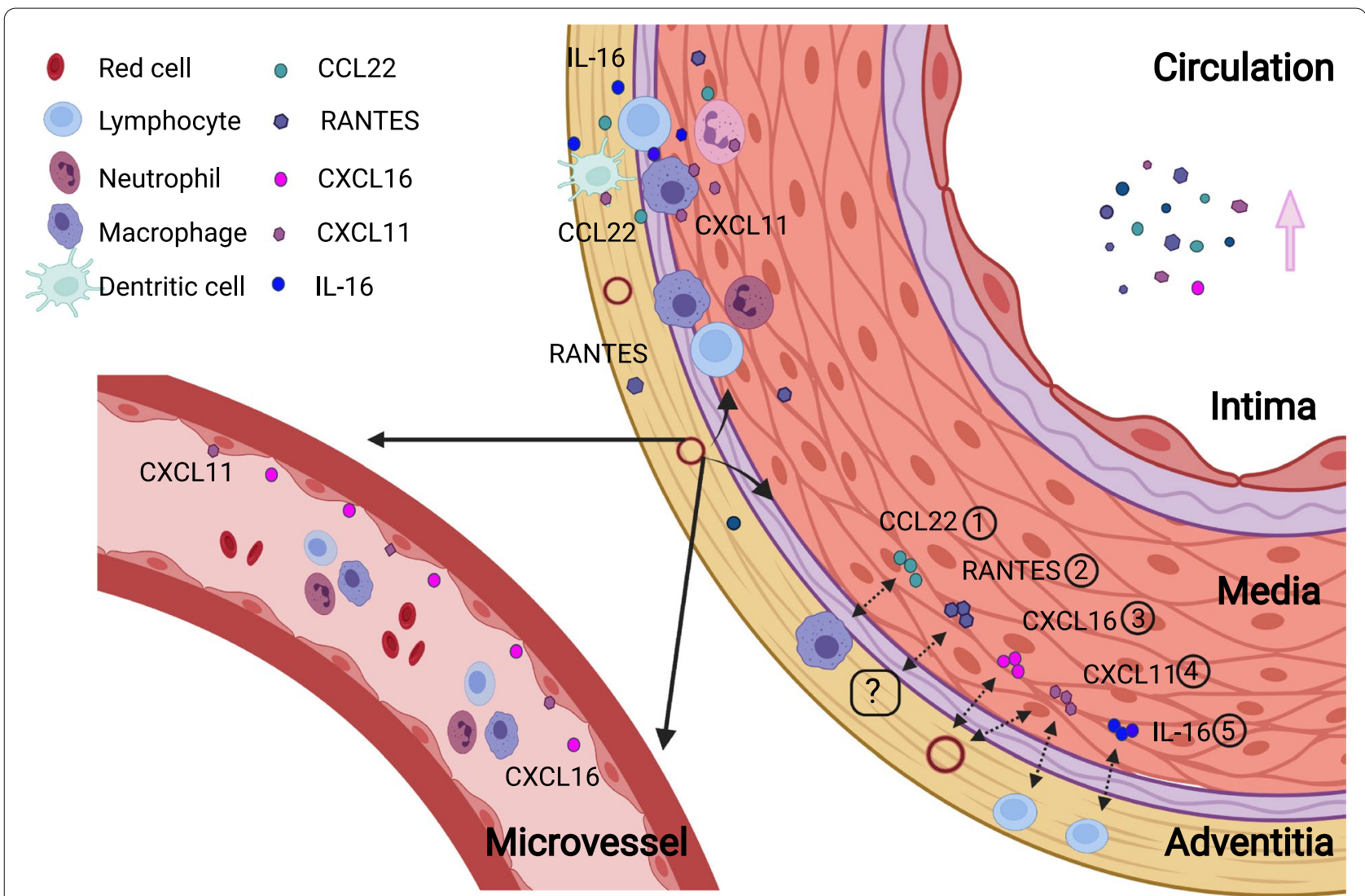

Fig. 4 A proposed model illustrating chemokines discovered in the present study and their potential role in the pathogenesis of TAK. CCL22, RANTES, CXCL16, CXCL11, and IL-16 were increased in the peripheral blood as well as vascular tissue of TAK. In active vascular lesions, the infiltration was predominated by $\mathrm{CD}^{+} \mathrm{T}$ cells, a less proportion of $\mathrm{CD} 68^{+}$macrophage and CD19+ B cells. Among these five chemokines, CCL22, IL-16, and CXCL11 were distributed in vascular infiltration. RANTES was expressed in a relative low level. In addition, CXCL11 was also expressed in adventitial microvessels, while CXCL16 was mainly expressed in adventitial microvessel wall. Based on their functions, their potential roles in TAK were presumed as followed: (1) CCL22 may participate in macrophage recruitment; (2) RANTES is able to recruit multiple cells, but the specific cell type it functions needs further exploration; (3) CXCL16 probably involves in migration of peripheral immune cells from adventitial microvessels to vascular lesions; (4) CXCL11 may recruit active T cells as well as participate in the recruitment immune cells from microvessels; (5) IL-16 probably promotes $\mathrm{CD}^{+} \mathrm{T}$ cells infiltration

a persistent high levels. Thus, CCL22, CXCL11, and IL-16 may participate in the vascular chronic inflammation or fibrotic process of TAK.

The key functions of the five TAK-related chemokines, namely, CCL22, RANTES, CXCL16, CXCL11, and IL-16, identified in this study are reviewed in Table 2. CCL22 is mainly produced by macrophages, dendritic cells, and can recruit macrophages via $\mathrm{CC}$ chemokine receptor 4 $[15,19]$. It has been implicated in inflammatory, allergic, as well as fibrotic disorders [24, 32-34]. In TAK, macrophage was a major cell type in vascular lesions [10]. Thus, CCL22 may be mainly derived from macrophages and involved in macrophage recruitment. CCL22 is also a biomarker of M2 macrophages [43]. It has been reported that M2 macrophages dominated M1 macrophages in TAK vascular lesions [44]. Our previous study also found macrophages experienced a M1 to M2 shift from untreated to treated vascular lesions [10]. This probably was an important reason for persistent high serum CCL22 levels in TAK. However, the relationship between CCL22 and vascular macrophages needs further verification.

RANTES is an inflammatory chemokine that exhibits chemotaxis towards multiple inflammatory cells, especially T cells via CCR $5[16,20]$. RANTES has been reported to play a role in multiple vascular diseases, tumors, and viral infections $[25,26,35,36]$. In the present study, the increase of serum RANTES is consistent with previous findings [7, 25]. However, its expression in vascular tissue has not ever been studied. In GCA, RANTES was co-localized with leucocytes in vascular 
adventitia [26], but this phenomenon was not prominent TAK vascular tissue according to the results in the present study. In the peripheral blood, a correlation was observed between RANTES and IL-6 in this study, which indirectly suggested its participation in the inflammatory process of TAK. Finally, the decrease in RANTES levels after treatment indicated a response to glucocorticoids and immunosuppressants treatment.

CXCL16 participates in various pathological processes such as immune cell recruitment, cell interactions, angiogenesis etc. $[17,21]$, leading to its involvement in multiple disorders [27, 28]. Importantly, CXCL16 has been reported to mediate the adhesion of platelets or leucocytes to endothelial cells [45]. In the present study, CXCL16 was mainly expressed in the adventitial microvessel wall. Therefore, CXCL16 probably plays a critical role in recruitment of immune cell from the peripheral blood to vascular lesions via adventitial microvessels. In addition, CXCL16 has been reported to play a pro-angiogenic role by recruiting endothelial progenitor cells [28]. Angiogenesis is also increased in vascular lesions in TAK, but whether CXCL16 also participates in neovascularization in TAK remains further investigation. Similar to RANTES, CXCL16 also can be downregulated in response to glucocorticoids and immunosuppressive treatment.

CXCL11 acts as a chemokine for $\mathrm{T}$ cells and macrophages via binding to the receptor CXCR3 [22]. The CXCL11/CXCR3 axis has been implicated in multiple autoimmune disorders and tumors [5, 22, 29, 37-39]. In GCA, CXCL11 expression was also observed in vascular lesions, and blocking IFN- $\gamma$ was found to reduce CXCL11 expression and further decrease macrophage infiltration [5]. In the present study, CXCL11 expression was mainly observed in microvessel wall and inflammatory lesions. This indicates its role in immune cell recruitment and activation in TAK. In the present study, persistently high levels of CXCL11 were found in the peripheral blood after glucocorticoids and immunosuppressive treatment, which may contributed to vascular chronic inflammation in TAK. Thus, it is worthy to explore whether CXCL11/ CXCR3 axis also involved in the pathogenesis of TAK in future studies.

IL-16 has chemotactic function in immune cells, especially $\mathrm{CD} 4^{+} \mathrm{T}$ cells $[18,23]$. Its expression was associated with tissue $\mathrm{CD}^{+}{ }^{+} \mathrm{T}$ lymphocyte infiltration in multiple disorders [46-48]. In antineutrophil cytoplasmic antibody-associated vasculitis, IL-16 was correlated with the vasculitis damage index [30] and was presumed to be involved in vascular fibrosis. In addition, it was reported to participate in the migration and invasion of vascular smooth muscle cells (VSMCs) [31]. In the present study, the positive correlation between IL-16 levels and CD4 ${ }^{+}$ $\mathrm{T}$ cell number and the close distribution of IL-16 to lymphocyte infiltrates also indicate an important role for IL-16 in $\mathrm{CD}^{+} \mathrm{T}$ cell recruitment. Further, a persistent high levels of IL-16 were observed in TAK after glucocorticoids and immunosuppressive treatment. Whether high levels of IL-16 contribute to chronic fibrosis in vascular lesions needs further exploration.

In the present study, although no correlations were observed between these chemokine levels and the disease activity markers ESR or CRP, high levels of IL-6 and IL-17 were detected along with the high levels of chemokines. This finding, combined with the close distribution of chemokines with vascular immune cells, implies that these chemokines may also be indicators of active immune responses in TAK. Additionally, the persistently high levels of CXCL11 and IL-16 after treatment probably indicates uncontrolled immune activation or their role in chronic vascular fibrosis. The correlations observed between CCL22, CXCL16, and IL-16 indicate that these chemokines may function in a synergistic way.

This study comprehensively investigated the chemokine profile of TAK and preliminarily explored the potential role of five identified chemokines in vascular immune cell recruitment. They may play different roles in vascular lesions by attracting different cell types. These findings provide important evidence for the pathogenesis of TAK, but there are also some limitations to this study. Firstly, in this study, four chemokines with lower levels screened from array had not been further validated. Lower levels of chemokines may reflect the usage of them by immune cells. Thus, it was worthy to have more research on them in future TAK studies. In addition, only five chemokines validated in the serum were detected in vascular tissue. Thus, certain critical chemokines in vascular tissue could have been overlooked due to inconsistencies between the peripheral blood and vascular tissue findings. Moreover, the treatment period was relatively short, so the effects of glucocorticoids and immunosuppressive agents on these chemokines need further verification Finally, the origins and roles of these chemokines remained to be explored further.

\section{Conclusions}

CCL22, RANTES, CXCL16, CXCL11, and IL-16 are identified as the major chemokines that may involve in the recruitment of immune cells in the vascular tissue of patients with TAK. Additionally, the persistently high levels of CCL22, CXCL11, and IL-16 observed after treatment might suggest their participation in vascular chronic inflammation or fibrosis and demonstrate the need for developing more efficacious treatment options. 


\section{Abbreviations}

TAK: Takayasu arteritis; GCA: Giant cell arteritis; ESR: Erythrocyte sedimentation rate; CRP: C-reactive protein; NK cells: Natural killer cells; ELISA: Enzyme-linked immunosorbent assay; VSMCs: Vascular smooth muscle cells.

\section{Supplementary Information}

The online version contains supplementary material available at https://doi. org/10.1186/s13075-022-02740-x.

Additional file 1: Supplementary Figure 1. Flow chart depicting the study design. Supplementary Figure 2. Gating strategies in flow cytometry for peripheral immune subsets analysis. Supplementary Figure 3. Results of chemokine array in the present study. Supplementary Figure 4. Vascular infiltration in patients with TAK. Supplementary Figure 5. Peripheral cytokine levels and their correlation with chemokine levels in patients with TAK. Supplementary Figure 6. Correlations between chemokines and disease activity markers in patients with TAK. Supplementary Figure 7. Correlations among different chemokines and changes in their levels after treatment. Supplementary Table 1. Clinical characteristics of the patients included for vascular tissue examination. Supplementary Table 2. Key functions of the five major chemokines.

\section{Acknowledgements}

Not applicable.

\section{Authors' contributions}

XFK was responsible for manuscript writing; SFW and XJD were responsible for immunohistochemical staining; WSY and Jinghua Wang were responsible for ELISA detection; YS and ZFJ were responsible for collection of clinical data; LYM, XMD, and HYC were responsible for patients' clinical follow-up; LLM was responsible for the statistical analysis; and LDJ was responsible for the research design. The author(s) read and approved the final manuscript.

\section{Funding}

This work was supported by the National Natural Science Foundation of China (grant numbers 82101888, 81771730, 81801598), by Shanghai Sailing Program (grant numbers 20YF1406800) the Science and Technology Commission of Shanghai Municipality (grant numbers 17140902000), the China Postdoctoral Science Foundation (grant number 2020M671008), the Clinical Research Project of Zhongshan Hospital, China (grant number 2020ZSLC14), and the Youth Research Fund of Zhongshan Hospital, Fudan University, China (grant number 2020ZYYS-001).

\section{Availability of data and materials}

The datasets used and/or analyzed during the current study are available from the corresponding author on reasonable request.

\section{Declarations}

\section{Ethics approval and consent to participate}

The study protocol was approved by the Ethics Committees of Zhongshan Hospital (B2016-168) and conformed to the ethical guidelines of the 1975 Declaration of Helsinki. Written informed consent was obtained from all the participants in this study.

\section{Consent for publication}

Not applicable.

\section{Competing interests}

The authors declare that they have no competing interests.

\section{Author details}

${ }^{1}$ Department of Rheumatology, Zhongshan Hospital Affiliated to Fudan University, Shanghai, China. ${ }^{2}$ Center of Clinical Epidemiology and Evidence-based Medicine, Fudan University, Shanghai, China.
Received: 6 September 2021 Accepted: 7 February 2022

Published online: 16 February 2022

\section{References}

1. Seyahi E. Takayasu arteritis: an update. Curr Opin Rheumatol. 2017;29(1):51-6.

2. Rutter M, Bowley J, Lanyon PC, Grainge MJ, Pearce FA. A systematic review and meta-analysis of the incidence rate of Takayasu arteritis. Rheumatology (Oxford). 2021;60(11):4982-90.

3. Matsumoto K, Suzuki K, Yoshimoto K, Seki N, Tsujimoto H, Chiba K, et al. Significant association between clinical characteristics and changes in peripheral immuno-phenotype in large vessel vasculitis. Arthritis Res Ther. 2019;21(1):304.

4. Kurata A, Saito A, Hashimoto H, Fujita K, Ohno SI, Kamma H, et al. Difference in immunohistochemical characteristics between Takayasu arteritis and giant cell arteritis: it may be better to distinguish them in the same age. Mod Rheumatol. 2019;29(6):992-1001.

5. Corbera-Bellalta M, Planas-Rigol E, Lozano E, Terrades-García N, Alba MA, Prieto-González $S$, et al. Blocking interferon $\gamma$ reduces expression of chemokines CXCL9, CXCL10 and CXCL11 and decreases macrophage infiltration in ex vivo cultured arteries from patients with giant cell arteritis. Ann Rheum Dis. 2016;75(6):1177-86.

6. Mahajan N, Dhawan V, Mahmood S, Malik S, Jain S. Extracellular matrix remodeling in Takayasu's arteritis: role of matrix metalloproteinases and adventitial inflammation. Arch Med Res. 2012;43(5):406-10.

7. Noris M, Daina E, Gamba S, Bonazzola S, Remuzzi G. Interleukin-6 and RANTES in Takayasu arteritis: a guide for therapeutic decisions? Circulation. 1999;100(1):55-60.

8. Dong H, Zhang Y, Zou Y, Chen Y, Yue J, Liu H, et al. Elevated chemokines concentration is associated with disease activity in Takayasu arteritis. Cytokine. 2021;143:155515.

9. Seko Y. Takayasu arteritis: insights into immunopathology. Jpn Heart J. 2000:41(1):15-26.

10. Kong X, Xu M, Cui X, Ma L, Cheng H, Hou J, et al. Potential role of macrophage phenotypes and CCL2 in the pathogenesis of Takayasu Arteritis. Front Immunol. 2021;12:646516.

11. Arend WP, Michel BA, Bloch DA, Hunder GG, Calabrese LH, Edworthy SM, et al. The American College of Rheumatology 1990 criteria for the classification of Takayasu arteritis. Arthritis Rheum. 1990;33(8):1129-34.

12. Hata A, Noda M, Moriwaki R, Numano F. Angiographic findings of Takayasu arteritis: new classification. Int J Cardiol. 1996;54(Suppl):S155-63.

13. Kerr GS, Hallahan CW, Giordano J, Leavitt RY, Fauci AS, Rottem M, et al. Takayasu arteritis. Ann Intern Med. 1994;120(11):919-29.

14. Kong $X$, Sun $Y, M a L$, Chen $H$, Wei L, Wu W, et al. The critical role of IL-6 in the pathogenesis of Takayasu arteritis. Clin Exp Rheumatol. 2016;34(3 Suppl 97):S21-7.

15. Yamashita $U$, Kuroda E. Regulation of macrophage-derived chemokine (MDC, CCL22) production. Crit Rev Immunol. 2002;22(2):105-14.

16. Marques RE, Guabiraba R, Russo RC, Teixeira MM. Targeting CCL5 in inflammation. Expert Opin Ther Targets. 2013;17(12):1439-60.

17. Zernecke A, Shagdarsuren E, Weber C. Chemokines in atherosclerosis: an update. Arterioscler Thromb Vasc Biol. 2008;28(11):1897-908.

18. Richmond J, Tuzova M, Cruikshank W, Center D. Regulation of cellular processes by interleukin-16 in homeostasis and cancer. J Cell Physiol. 2014;229(2):139-47.

19. Mantovani A, Gray PA, Van Damme J, Sozzani S. Macrophage-derived chemokine (MDC). J Leukoc Biol. 2000;68(3):400-4.

20. Suffee N, Richard B, Hlawaty H, Oudar O, Charnaux N, Sutton A. Angiogenic properties of the chemokine RANTES/CCL5. Biochem Soc Trans. 2011;39(6):1649-53.

21. Korbecki J, Bajdak-Rusinek K, Kupnicka P, Kapczuk P, Simińska D, Chlubek $D$, et al. The role of CXCL16 in the pathogenesis of cancer and other diseases. Int J Mol Sci. 2021;22(7):3490.

22. Tokunaga R, Zhang W, Naseem M, et al. CXCL9, CXCL10, CXCL11/CXCR3 axis for immune activation - a target for novel cancer therapy. Cancer Treat Rev. 2018;63:40-7. https://doi.org/10.1016/j.ctrv.2017.11.007. 
23. Cruikshank WW, Kornfeld H, Center DM. Interleukin-16. J Leukoc Biol. 2000;67(6):757-66.

24. Jakiela B, Sanak M, Szczeklik W, Sokolowska B, Plutecka H, Mastalerz L, et al. Both Th2 and Th17 responses are involved in the pathogenesis of Churg-Strauss syndrome. Clin Exp Rheumatol. 2011;29(1 Suppl 64):S23-34

25. Dhawan V, Mahajan N, Jain S. Role of C-C chemokines in Takayasu's arteritis disease. Int J Cardiol. 2006;112(1):105-11.

26. Brühl $H$, Vielhauer $V$, Weiss $M$, Mack M, Schlöndorff $D$, Segerer S. Expression of DARC, CXCR3 and CCR5 in giant cell arteritis. Rheumatology (Oxford). 2005;44(3):309-13.

27. Izquierdo MC, Martin-Cleary C, Fernandez-Fernandez B, Elewa U, Sanchez-Niño MD, Carrero JJ, et al. CXCL16 in kidney and cardiovascular injury. Cytokine Growth Factor Rev. 2014;25(3):317-25.

28. Isozaki T, Arbab AS, Haas CS, Amin MA, Arendt MD, Koch AE, et al. Evidence that CXCL16 is a potent mediator of angiogenesis and is involved in endothelial progenitor cell chemotaxis: studies in mice with $\mathrm{K} / \mathrm{BxN}$ serum-induced arthritis. Arthritis Rheum. 2013;65(7):1736-46. https://doi. org/10.1002/art.37981.

29. Antonelli A, Ferri C, Ferrari SM, Ruffilli I, Colaci M, Frascerra S, et al. High serum levels of CXCL11 in mixed cryoglobulinemia are associated with increased circulating levels of interferon- $\gamma$. J Rheumatol. 2011;38(9):1947-52

30. Yoon T, Pyo JY, Ahn SS, Song JJ, Park YB, Lee SW. Serum interleukin-16 significantly correlates with the Vasculitis Damage Index in antineutrophil cytoplasmic antibody-associated vasculitis. Arthritis Res Ther. 2020;22(1):73

31. Park SL, Hwang B, Lee SY, Kim WT, Choi YH, Chang YC, et al. p21WAF1 is required for interleukin-16-induced migration and invasion of vascular smooth muscle cells via the p38MAPK/Sp-1/MMP-9 pathway. PLoS One. 2015:10(11):e0142153.

32. Dogan RN, Long N, Forde E, Dennis K, Kohm AP, Miller SD, et al. CCL22 regulates experimental autoimmune encephalomyelitis by controlling inflammatory macrophage accumulation and effector function. J Leukoc Biol. 2011:89(1):93-104.

33. Yoshie O, Matsushima K. CCR4 and its ligands: from bench to bedside. Int Immunol. 2015;27(1):11-20.

34. Inoue T, Fujishima S, Ikeda E, Yoshie O, Tsukamoto N, Aiso S, et al. CCL22 and CCL17 in rat radiation pneumonitis and in human idiopathic pulmonary fibrosis. Eur Respir J. 2004;24(1):49-56.

35. Lapteva N, Huang XF. CCL5 as an adjuvant for cancer immunotherapy. Expert Opin Biol Ther. 2010;10(5):725-33.

36. Crawford A, Angelosanto JM, Nadwodny KL, Blackburn SD, Wherry EJ. A role for the chemokine RANTES in regulating CD8 T cell responses during chronic viral infection. PLoS Pathog. 2011;7(7):e1002098.

37. Burdick MD, Murray LA, Keane MP, Xue YY, Zisman DA, Belperio JA, et al. CXCL11 attenuates bleomycin-induced pulmonary fibrosis via inhibition of vascular remodeling. Am J Respir Crit Care Med. 2005;171(3):261-8.

38. Antonelli A, Ferrari SM, Frascerra S, Di Domenicantonio A, Nicolini A Ferrari $P$, et al. Increase of circulating CXCL9 and CXCL11 associated with euthyroid or subclinically hypothyroid autoimmune thyroiditis. J Clin Endocrinol Metab. 2011;96(6):1859-63.

39. Zohar Y, Wildbaum G, Novak R, Salzman AL, Thelen M, Alon R, et al. CXCL11-dependent induction of FOXP3-negative regulatory T cells suppresses autoimmune encephalomyelitis. J Clin Invest. 2014;124(5):2009-22.

40. Lee S, Kaneko H, Sekigawa I, Tokano Y, Takasaki Y, Hashimoto H. Circulating interleukin-16 in systemic lupus erythematosus. Br J Rheumatol. 1998;37(12):1334-7.

41. Cho ML, Jung YO, Kim KW, Park MK, Oh HJ, Ju JH, et al. IL-17 induces the production of IL-16 in rheumatoid arthritis. Exp Mol Med. 2008:40(2):237-45.

42. Murota A, Suzuki K, Kassai Y, Miyazaki T, Morita R, Kondo Y, et al. Serum proteomic analysis identifies interleukin 16 as a biomarker for clinical response during early treatment of rheumatoid arthritis. Cytokine. 2016:78:87-93.

43. Cui X, Kong $X$, Chen R, Ma L, Jiang L. The potential role of leflunomide in inhibiting vascular fibrosis by down-regulating type-II macrophages in Takayasu's arteritis. Clin Exp Rheumatol. 2020;38 Suppl 124(2):69-78.
44. Dos Santos JP, Artigiani Neto R, Mangueira CLP, Filippi RZ, Gutierrez PS, Westra J, et al. Associations between clinical features and therapy with macrophage subpopulations and T cells in inflammatory lesions in the aorta from patients with Takayasu arteritis. Clin Exp Immunol. 2020;202(3):384-93.

45. Collado A, Marques P, Escudero P, Rius C, Domingo E, Martinez-Hervás $S$, et al. Functional role of endothelial CXCL16/CXCR6-platelet-leucocyte axis in angiotensin II-associated metabolic disorders. Cardiovasc Res. 2018;114(13):1764-75.

46. Laberge S, Ghaffar O, Boguniewicz M, Center DM, Leung DY, Hamid Q. Association of increased CD4+T-cell infiltration with increased IL-16 gene expression in atopic dermatitis. J Allergy Clin Immunol. 1998;102(4 Pt 1):645-50.

47. Skundric DS, Cruikshank WW, Drulovic J. Role of IL-16 in CD4+T cellmediated regulation of relapsing multiple sclerosis. J Neuroinflammation. 2015;12:78.

48. Stabile E, Kinnaird T, la Sala A, Hanson SK, Watkins C, Campia U, et al. CD8+ T lymphocytes regulate the arteriogenic response to ischemia by infiltrating the site of collateral vessel development and recruiting CD4+ mononuclear cells through the expression of interleukin-16. Circulation 2006;113(1):118-24.

\section{Publisher's Note}

Springer Nature remains neutral with regard to jurisdictional claims in published maps and institutional affiliations.

Ready to submit your research? Choose BMC and benefit from:

- fast, convenient online submission

- thorough peer review by experienced researchers in your field

- rapid publication on acceptance

- support for research data, including large and complex data types

- gold Open Access which fosters wider collaboration and increased citations

- maximum visibility for your research: over $100 \mathrm{M}$ website views per year

At BMC, research is always in progress.

Learn more biomedcentral.com/submissions 